\title{
An improved tracking model based on self-adaptive Kalman filter
}

\author{
Meng LIAN ${ }^{1, a}$, Yi SUN* \\ 1, *Beijing University of Posts and Telecommunications, 100876, China \\ a mail: lianmeng@bupt.edu.cn, *mail: sunyiisse@bupt.edu.cn
}

Keywords: Kalman filtering, Self-adaptive, Irestrictions,Multi-model,VSIFT map

\begin{abstract}
The self-adaptive Kalman filter algorithm can solve the problem of filtering divergence and tracking in conventional Kalman filter algorithm, so it is widely used in GPS data processing of vehicles and ships. But in practical application, the tracking of adaptive algorithm is still insufficient. Therefore, this paper proposes an improved tracking model based on adaptive Kalman filter. In this paper, by adding velocity and acceleration constraints to the conventional Kalman equation, we find that the equation only changes in the one-step prediction equation. By adding the changing parameters to the self-adaptive parameters in self-adaptive Kalman filter algorithm, it can effectively enhance the tracking ability of the adaptive algorithm to the velocity and acceleration changes. At last, the improved model is improved by multi-model and VSIFT. The simulation results show that the improved method can effectively enhance the model tracking performance.
\end{abstract}

\section{Introduction}

In order to improve the tracking ability of self-adaptive Kalman filter, this paper improves the adaptive algorithm by increasing the speed constraints, acceleration constraints and combining with the multi-model probabilistic problem to increase the tracking performance and reduce the computational complexity.

\section{Self-adaptive Kalman filter}

As the normal Kalman filter is only suitable for linear systems and there are poor robustness, lack of tracking and other issues, so people put forward the self- adaptive Kalman filter.In order to make the filter has excellent performance, self- adaptive Kalman filter mainly uses time-varying fading factor on the past data fading to weakening the old data on the current filter value.The algorithmic process is represented by the function[1]:

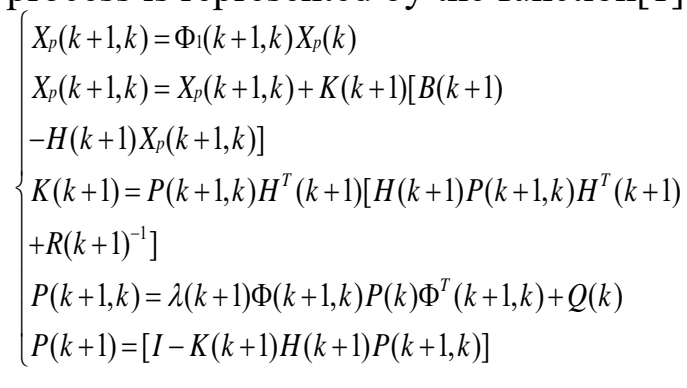

In the function,

$$
\begin{aligned}
& \lambda(k+1)=\max \{1, \operatorname{tr}\{N(k+1)] / \operatorname{tr}[M(k+1)]\} \\
& \mathrm{M}(\mathrm{k}+1)=H(k+1) \Phi(k+1, k) P(k) \Phi^{T}(k+1, k) H^{T}(k+1) \\
& N(k+1)=C_{0}(k+1)-H(k+1) Q(k+1) H^{T}(k+1)-R(k+1) \\
& C_{0}(k+1)=\left\{\begin{array}{c}
\lambda(k) v(k+1) v^{T}(k+1) / 1+\lambda(k), k>1 \\
v(1) v^{T}(1) / 2, k=0
\end{array}\right. \\
& v(k+1)=L(k+1) X_{p}(k+1, k)
\end{aligned}
$$

$\operatorname{tr}[\cdot]$ is the trace of the matrix, $X_{\mathrm{p}}$ is the system state, $\mathrm{X}_{\mathrm{p}}(\mathrm{k})$ is the best estimate of the state at time $\mathrm{k}$, $\mathrm{X}_{\mathrm{p}}(\mathrm{k}+1, \mathrm{k})$ is the predicted value calculated at time $\mathrm{k}+1$ base on $\mathrm{X}_{\mathrm{p}}(\mathrm{k}), \mathrm{P}$ is the covariance of $\mathrm{X}_{\mathrm{p}} . \Phi$ is the system state transition matrix, $\mathrm{K}$ is the Kalman gain matrix, $\mathrm{B}$ is the system measurement, $\mathrm{R}$ is the covariance of the measured noise, $\mathrm{Q}$ is the covariance of system noise. 


\section{Improved Tracking Model of Self-adaptive Kalman filter}

The GPS receiver can output not only the position result but also the velocity information [2] and the acceleration information. We can improve the positioning accuracy and increase model tracking if the speed and acceleration information are joined the Kalman equation as constraints. So, the adaptive Kalman equation is modified.

State vector $X(k)=\left(x, \dot{x} \ddot{x}, y, \dot{y}, \ddot{y}, \varepsilon_{x}, \varepsilon_{y}\right), \varepsilon$ is the receiver position error. There are:

$$
\varepsilon(k+1)=\frac{2 \tau-1}{2 \tau+1} \varepsilon(k)+\frac{2 \tau-1}{2 \tau+1} w_{x, y}
$$

State function: $X(k+1)=\Phi(k+1, k) X(k)+\Gamma W(k-1)$

$$
\Phi(k+1, k)=\left[\begin{array}{cccccccc}
1 & T & \frac{1}{2} T^{2} & 0 & 0 & 0 & 0 & 0 \\
0 & 1 & T & 0 & 0 & 0 & 0 & 0 \\
0 & 0 & 1 & 0 & 0 & 0 & 0 & 0 \\
0 & 0 & 0 & 1 & T & \frac{1}{2} T^{2} & 0 & 0 \\
0 & 0 & 0 & 0 & 1 & 0 & 0 & 0 \\
0 & 0 & 0 & 0 & 0 & 1 & 0 & 0 \\
0 & 0 & 0 & 0 & 0 & 0 & \frac{2 \tau-1}{2 \tau+1} & 0 \\
0 & 0 & 0 & 0 & 0 & 0 & 0 & \frac{2 \tau-1}{2 \tau+1}
\end{array}\right]
$$

$$
W(n)=\left[\dot{\delta} \dot{X}, \dot{\delta Y}, w_{x}, w_{y}\right]
$$

$\delta \ddot{X}, \delta \ddot{Y}$ are the movement carrier velocity error interference; $\mathrm{w}_{\mathrm{x}}, \mathrm{w}_{\mathrm{y}}$ are the driving noise of the position deviation.Noise matrix $\Gamma$ is:

$$
\Gamma=\left[\begin{array}{cccc}
0 & 0 & 0 & 0 \\
T & 0 & 0 & 0 \\
1 & 0 & 0 & 0 \\
0 & 0 & 0 & 0 \\
0 & T & 0 & 0 \\
0 & 1 & 0 & 0 \\
0 & 0 & \frac{\tau}{2 \tau+1} & 0 \\
0 & 0 & 0 & \frac{\tau}{2 \tau+1}
\end{array}\right]
$$

$$
\begin{aligned}
& Q(k)=\left[\begin{array}{cccc}
\sigma_{\ddot{X}}^{2} & 0 & 0 & 0 \\
0 & \sigma_{\ddot{Y}}^{2} & 0 & 0 \\
0 & 0 & \sigma_{\varepsilon x}^{2} & 0 \\
0 & 0 & 0 & \sigma_{\varepsilon y}^{2}
\end{array}\right] \\
& Z(k)=\left(x_{r} v_{x}, y_{r}, v_{y}\right)
\end{aligned}
$$

GPS output the result $\left(\mathrm{x}_{\mathrm{r}}, \mathrm{y}_{\mathrm{r}}\right)$ includes real variables $(x, y)$, first order Markova errors ${ }^{\left(\varepsilon_{x}, \varepsilon_{y}\right)}$ and measurement error ${ }^{\left(\varepsilon_{L x}, \varepsilon_{L y}\right)}$.

$$
\begin{aligned}
& X_{r}=X+\varepsilon_{X}+\varepsilon_{L x} \\
& y_{r}=X+\varepsilon_{y}+\varepsilon_{L y}
\end{aligned}
$$

Observation matrix $\mathrm{H}$;

$$
H=\left[\begin{array}{llllllll}
1 & 0 & 0 & 0 & 0 & 0 & 1 & 0 \\
0 & 1 & 0 & 0 & 0 & 0 & 0 & 0 \\
0 & 0 & 0 & 1 & 0 & 0 & 0 & 1 \\
0 & 0 & 0 & 0 & 1 & 0 & 0 & 0
\end{array}\right]
$$


The location of the vehicle at time $t_{n}$ is $\left(X_{n}, Y_{n}\right),\left(X_{n-1}, Y_{n-1}\right)$ at $t_{n-1}$, the relationship between Vehicle speed, acceleration and position is as follows:

$V_{x}=\frac{X_{n}-X_{n-1}}{t_{n}-t_{n-1}}, V_{y}=\frac{Y_{n}-Y_{n-1}}{t_{n}-t_{n-1}}$

Write in the form of matrix:

$\operatorname{NMTX}(k+1)=X(k)$

$\mathrm{M}, \mathrm{N}, \mathrm{T}$ are the speed transfer matrix, acceleration transfer matrix and time matrix are:

$$
M=\left[\begin{array}{cccccccc}
1 & -1 & 0 & 0 & 0 & 0 & 0 & 0 \\
0 & 1 & 0 & 0 & 0 & 0 & 0 & 0 \\
0 & 0 & 1 & 0 & 0 & 0 & 0 & 0 \\
0 & 0 & 0 & 1 & -1 & 0 & 0 & 0 \\
0 & 0 & 0 & 0 & 1 & 0 & 0 & 0 \\
0 & 0 & 0 & 0 & 0 & 1 & 0 & 0 \\
0 & 0 & 0 & 0 & 0 & 0 & 1 & 0 \\
0 & 0 & 0 & 0 & 0 & 0 & 0 & 1
\end{array}\right]
$$

(3.12) $N=\left[\begin{array}{rrrrrrrr}1 & 0 & -\frac{1}{2} & 0 & 0 & 0 & 0 & 0 \\ 0 & 1 & -1 & 0 & 0 & 0 & 0 & 0 \\ 0 & 0 & 1 & 0 & 0 & 0 & 0 & 0 \\ 0 & 0 & 0 & 1 & 0 & -\frac{1}{2} & 0 & 0 \\ 0 & 0 & 0 & 0 & 1 & -1 & 0 & 0 \\ 0 & 0 & 0 & 0 & 0 & 1 & 0 & 0 \\ 0 & 0 & 0 & 0 & 0 & 0 & 1 & 0 \\ 0 & 0 & 0 & 0 & 0 & 0 & 0 & 1\end{array}\right]$

$(3.13)_{T}=\left[\begin{array}{cccccccc}1 & T & T^{2} & 0 & 0 & 0 & 0 & 0 \\ 0 & 1 & T & 0 & 0 & 0 & 0 & 0 \\ 0 & 0 & 1 & 0 & 0 & 0 & 0 & 0 \\ 0 & 0 & 0 & 1 & T & T^{2} & 0 & 0 \\ 0 & 0 & 0 & 0 & 1 & T & 0 & 0 \\ 0 & 0 & 0 & 0 & 0 & 1 & 0 & 0 \\ 0 & 0 & 0 & 0 & 0 & 0 & 1 & 0 \\ 0 & 0 & 0 & 0 & 0 & 0 & 0 & 1\end{array}\right]$

Connect the constraint condition and the system equation:

$$
\left\{\begin{array}{c}
X(k+1)=\Phi(k+1, k) X(k)+W(k+1) \\
Z(k+1)=H(k+1) X(k+1)+V(k+1) \\
N M T X(k+1)=X(k)
\end{array}\right.
$$

Because the constraint function is introduced into the model, to satisfy the new conditional relation between the state parameters, the Kalman filter with speed and acceleration constraints need be deduced with the original state parameters based on the principle of minimum variance estimation. The minimum variance estimate satisfies:

$$
\begin{aligned}
& \min E\left(\|X-X\|^{2} \mid Z\right) \\
& E\left(\|X-X\|^{2} \mid Z\right) \\
& =\sum(X(k+1)-\bar{X}(k+1))^{T}(X(k+1)-\bar{X}(k+1)) P(X(k+1) / Z) \\
& =\sum X^{T}(k+1) X(k+1) P(X(k+1) / Z) \\
& -2 \bar{X}^{T}(k+1) \sum X(k+1) P(X(k+1) / Z)+X^{T}(k+1) X(k+1) \\
& =\sum X^{T}(k+1) X(k+1) P(X(k+1) / Z)-2 \bar{X}^{T}(k+1) X_{p}(k+1) \\
& +X^{T}(k+1) X(k+1)
\end{aligned}
$$

$$
X_{p}(k+1)=\sum X(n) P(X(k+1) / Z)
$$

Then establish the Lagrange equation and determine partial derivative of the equation on both sides of the $\mathrm{X}$ and $\Lambda$ :

$$
\begin{aligned}
& L=E\left(\|X-X\|^{2} \mid Z\right)+2 \Lambda\left(N M T \bar{X}(k+1)-X_{p}(k)\right) \\
& =\sum X^{T}(k+1) X(k+1) P(X(k+1) / Z)-2 \bar{X}^{T}(k+1) X_{p}(k+1) \\
& +X^{T}(k+1) X(k+1)+2 \Lambda\left(N M T \bar{X}(k+1)-X_{p}(k)\right)
\end{aligned}
$$

$$
\left\{\begin{array}{c}
\frac{\partial L}{\partial X}=-2 X_{p}(k+1)+2 \bar{X}(k+1)+2 N M T \Lambda=0 \\
\frac{\partial \Lambda}{\partial X}=N M T \bar{X}(k+1)-X_{p}(k)=0
\end{array}\right.
$$




$$
\left\{\begin{array}{c}
\Lambda=\frac{N M T X_{p}(k+1)-X_{p}(k)}{N N^{T} M M^{T} T T^{T}} \\
\bar{X}(k+1)=X_{p}(k+1)-N M T \frac{N M T X_{p}(k+1)-X_{p}(k)}{N N^{T} M M^{T} T T^{T}}
\end{array}\right.
$$

After joining speed and acceleration constraints, the only difference is that one-step prediction value is added with a correction term related to the constraint condition $N M T \frac{N M T X_{p}(k+1, k)-X_{p}(k)}{M M^{T} N N^{T} T T^{T}}$. This correction item contains velocity and acceleration information. When the kinematic state of the carrier is abruptly changed, the correction term also increases. Therefore, the adaptive coefficient $\lambda(\mathrm{k}+1)$ of the self-adaptive Kalman filter can be adjusted according to the correction term, thereby improving the adaptive Kalman filter.

The size of $\lambda(\mathrm{k}+1)$ depends on $\mathrm{N}(\mathrm{k}+1)$ to a great extent [3]. After introducing the adaptive adjustment, the expression of $\mathrm{N}(\mathrm{k}+1)$ is:

$$
N(k+1)=C_{0}(k+1)-H(k+1) Q(k) H^{T}(k+1)-R(k+1)+D
$$

When the carrier movement is normal, $\mathrm{D}=0$; when the state changes, $D=\left(N M T \frac{N M T X_{p}(k+1, k)-X_{p}(k)}{M M^{T} N N^{T} T T^{T}}\right)^{2} \eta$

$$
M=\left[\begin{array}{cccccc}
1 & -1 & 0 & 0 & 0 & 0 \\
0 & 1 & 0 & 0 & 0 & 0 \\
0 & 0 & 1 & 0 & 0 & 0 \\
0 & 0 & 0 & 1 & -1 & 0 \\
0 & 0 & 0 & 0 & 1 & 0 \\
0 & 0 & 0 & 0 & 0 & 1
\end{array}\right](3.24) N=\left[\begin{array}{rrrrrr}
1 & 0 & -\frac{1}{2} & 0 & 0 & 0 \\
0 & 1 & -1 & 0 & 0 & 0 \\
0 & 0 & 1 & 0 & 0 & 0 \\
0 & 0 & 0 & 1 & 0 & -\frac{1}{2} \\
0 & 0 & 0 & 0 & 1 & -1 \\
0 & 0 & 0 & 0 & 0 & 1
\end{array}\right] \text { (3.25) } T=\left[\begin{array}{cccccc}
1 & T & T^{2} & 0 & 0 & 0 \\
0 & 1 & T & 0 & 0 & 0 \\
0 & 0 & 1 & 0 & 0 & 0 \\
0 & 0 & 0 & 1 & T & T^{2} \\
0 & 0 & 0 & 0 & 1 & T \\
0 & 0 & 0 & 0 & 0 & 1
\end{array}\right] \text { (3.26) }
$$

$\eta$ is the adjustment parameters.

In order to monitor the motion state, this paper introduces the multi-model method. The multi-model method is mainly applied to the state estimation of the system with time-varying characteristics. It is particularly applicable to maneuvering target tracking. In the multi-model method, a finite number of target models are assumed and each model corresponds to different maneuvering input levels. After calculating posterior probability of each model as the correct, we can give the final target state estimation by weighting the state estimation of each model correctly [4]. The weighting factor is the correct posterior probability of the model.

Assuming there are $\mathrm{r}$ models, there is a model update probability for $\mathrm{j}=1,2, \ldots$, $u^{j}{ }_{k+1}=P\left\{M_{k+1}=M_{j} / Z(k+1)\right\}$

$$
\begin{aligned}
\mathrm{r} & =\frac{1}{c} \Lambda^{j}{ }_{k+1} \sum_{i=1}^{r} P\left\{M_{k+1}=M_{j} / M_{k}=M_{i}, Z_{k}\right\} P\left\{M_{i} / Z_{k}\right\} \\
& =\frac{1}{C} \Lambda^{j}{ }_{k+1} \sum_{i=1}^{r} p_{i j} u_{k}=\frac{1}{C} \Lambda^{j}{ }_{k+1} \bar{C}_{j}
\end{aligned}
$$

$$
\begin{aligned}
& \Lambda^{j}(k+1)=N\left(Z(k+1) ; Z^{j}(k+1, k), S^{j}(k+1)\right) \\
& =\frac{1}{\sqrt{2 \pi S^{j}(k+1)}} \exp \left\{-\frac{1}{2}\left[Z(k+1)-Z_{p}^{j}(k+1, k)\right]^{T}\left(S^{j}(k+1)\right)^{-1}\left[Z(k+1)-Z_{p}^{j}(k+1, k)\right]\right\}
\end{aligned}
$$


$C=\sum_{i=1}^{r} \Lambda_{k+1}^{j} \bar{C}_{j}$

When the carrier movement state changes abruptly, the carrier model changes and the model updates, so the variance of model update probability becomes smaller. Thus, the state of motion can be monitored with the variance of the model update probability.

$$
\text { Thus, } D=\left\{\begin{array}{cc}
0 & , E\left\{\left[u-u^{2}(k)\right\}<R\right. \\
\left(N M T \frac{N M T X_{p}(k+1, k)-X_{p}(k)}{M M^{T} N N^{T} T T^{T}}\right)^{2} \eta & , E\left\{\left[u-u^{2}(k)\right\} \geq R\right.
\end{array}\right.
$$

$\mathrm{R}$ is selected according to the actual desired accuracy.

\section{Error Analysis of GPS Sampling}

Although the GPS sampling interval is theoretically $1 \mathrm{~s}$, the sampling interval may be not accurate enough $\mathrm{n}$ practical applications. So, this paper improves VSIFT [5] map to detect the sampling interval.

Suppose there are $\mathrm{t} 1, \mathrm{t} 2, \ldots, \mathrm{tn}, \mathrm{n}$ times sampling, so there are $(\mathrm{t} 2-\mathrm{t} 1),(\mathrm{t} 3-\mathrm{t} 2), \ldots,(\mathrm{tn}-\mathrm{tn}-1), \mathrm{n}-1$ sampling intervals, denoted as $\mathrm{x} 1, \mathrm{x} 2, \ldots \mathrm{xn}$, x follow a normal distribution. Now, let us consider the process running from $\mathrm{t} t 0=0$. Before that, the process has been operating in a controlled state for a period of time and the estimated values $\mu$ e and $\sigma \mathrm{e} 2$ of the sampling interval mean $\mu$ and variance $\sigma 2$ are obtained. It is assumed that in order to obtain these two estimates, $\mathrm{N}+1$ samples are taken in advance for the process in the controlled state to obtain N GPS sampling intervals.

Then, as long as the process does not display an exception, the process samples as the time interval $\Delta t$. However, if a sample indicates an anomaly in the process, that is, there will be a change in the GPS sampling interval and additional samples will be added between these fixed points in time. Assuming that the time interval between the fixed sampling points is bisected into $\mu$ subintervals of length $\mathrm{d} 1$ such that $\mathrm{d} 1,2 \mathrm{~d} 1,3 \mathrm{~d} 1, \ldots,(\mu-1) \mathrm{d} 1$ occur in the interior. There is a total of $\mu-1$ possible samples for example and $\mu-1$ additional sample time intervals are generated.

In order to determine the circumstances under which additional samples need to be added, the region between the control limits may be divided into regions D1 and D2.

$$
\begin{aligned}
& D_{1}=\left[\mu_{0}-h \sigma_{\mathrm{e}}, \mu_{0}-g \sigma_{\mathrm{e}}\right] \mathrm{U}\left[\mu_{0}+h \sigma_{\mathrm{e},}, \mu_{0}+g \sigma_{\mathrm{e}}\right] \\
& D_{2}=\left[\mu_{0}-h \sigma_{\mathrm{e}}, \mu_{0}+g \sigma_{\mathrm{e}}\right]
\end{aligned}
$$

Where $0<\mathrm{g}<\mathrm{h}$.

For the Kth sampling, the decision rules are as follows:

I. If the sampling interval Xk exceeds the control limit, a process anomaly signal is issued;

II. If $\mathrm{Xk} \in$ then the times $\mathrm{d} 1$ and $2 \mathrm{~d} 1$ are taken for the $\mathrm{k}+1$ and $\mathrm{k}+2$ samples and the sampling interval $\mathrm{x}_{\mathrm{k}+1}$ is calculated;

III. If $x_{k+1} \in \frac{1}{d_{1}} D_{2}$, then the next sampling time is the next fixed sampling time.

After processing of the GPS output signal through the VSIFT map method, $T=1$ when no process error signal is issued and $\mathrm{T}=\frac{1}{d_{1}}$ when a process abnormality signal is issued.

Through the time error processing, GPS sampling time error of the speed factors reduced to $\frac{1}{d_{1}}$, the acceleration factor reduced to $\left(\frac{1}{d_{1}}\right)^{2}$. 


\section{Summary}

In improved Self-adaptive Kalman filter model,

$$
\begin{gathered}
N(k+1)=C_{0}(k+1)-H(k+1) Q(k) H^{T}(k+1)-R(k+1)+D \\
D=\left\{\begin{array}{cc}
0 & , E\left\{\left[u-u^{2}(k)\right\}<R\right. \\
\left(N M T \frac{N M T X_{p}(k+1, k)-X_{p}(k)}{M M^{T} N N^{T} T T^{T}}\right)^{2} \eta & , E\left\{\left[u-u^{2}(k)\right\} \geq R\right.
\end{array}\right.
\end{gathered}
$$

\section{Model Simulations}

Assuming that the initial position of an object on the plane is $(0,30)$, its initial velocity in the $\mathrm{x}$-axis direction is $0.1 \mathrm{~m} / \mathrm{s}$ and the initial velocity on the y-axis is also $0.1 \mathrm{~m} / \mathrm{s}$ and do irregular motion in the plane (in the $\mathrm{x}, \mathrm{y}$ direction, respectively, by adding the acceleration of the noise). The trajectory is known amount. Another two reconnaissance aircraft can independently measure the real-time distance between them and the object.The following Kalman filter model is established:

$X(k+1)=\Phi(k+1, k) X(k)+B(t) u(k+1)+W(k+1)$

$Z(k+1)=H(k+1) X(k+1)+V(k+1)$

The Kalman equation is used to filter the motion trajectory of the object. The system noise and the measurement noise of the Kalman equation are independently uncorrelated Gaussian white noise. $\mathrm{T}$ is to follow the mean 1 variance 0.05 of the normal distribution of random numbers. The filtering starts at the third epoch and the whole process lasts 300 s to get the object's trajectory. Take $\boldsymbol{\eta}=1, \mathrm{R}$ $=0.06, \mathrm{~d} 1=3$ to complete the improved model simulation.

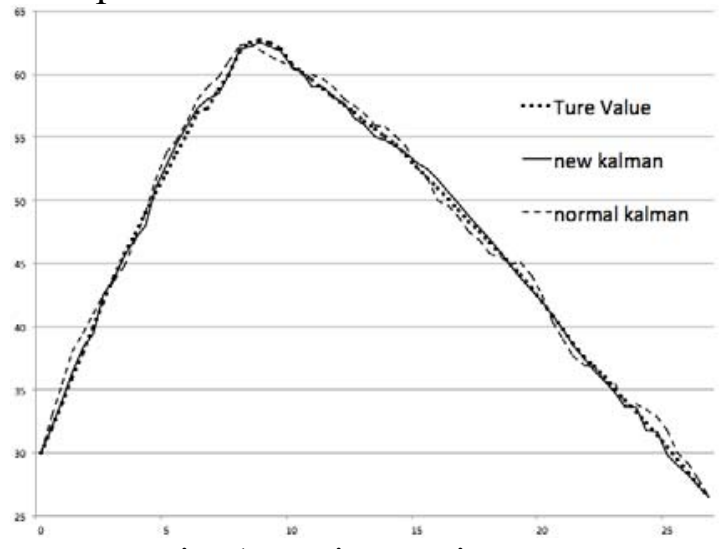

Fig. 1 Motion Trajectory Map

It can be seen that the improved model has better tracking performance after the carrier motion state is mutated.

\section{Acknowledgements}

This work was financially supported by the Research Innovation Fund for College Students of Beijing University of Posts and Telecommunications.

\section{References}

[1] Hu Guo-rong, Ou Jikun. An Improved Kalman Filtering Algorithm for High Dynamic GPS Positioning [N]. ACTA GEODAETICA ET CARTOGRAPHICA SINICA. 1999, 28(4).

[2] Liu Qiangang.GPS Positioning Algorithm and Its Application in Intelligent Public Transport[D]. Hunan University. 2009

[3] Lei Liping. Research on GPS Dynamic Kalman Filtering Algorithm [D]. University of Electronic Science and Technology of China.2003 
[4] Dong Wenli. Research on Kalman Filtering Algorithm in Vehicle Location and Navigation[D]. Henan. Zhengzhou University. 2007.

[5] Liu Jianbin, Zhang Weiming. Mean and Range Control Charts for Variable Sampling Intervals Sampled at Fixed Time [N]. JOURNAL OF ZHEJIANG INSTITUTE OF SCIENCE AND TECHNOLOGY. 2001, 18(4). 